\title{
Los seminarios apoyados con formación online como estrategia de aprendizaje autónomo en últimos cursos de Máster: caso práctico en el MUIHMA-UPV
}

\author{
López-Jiménez, P. Amparo; Pérez-Sánchez, Modesto \\ Departamento de Ingeniería Hidráulica y Medio Ambiente. Universitat Politècnica de València.
}

\begin{abstract}
The seminars development in the Master's Degree in Hydraulic Engineering and Environment MUIHMA contributes to a complementary training transversal to the speciality that the student develops. The intensive nature in terms of time of training means that the teacher must consider, will active methodologies of training by students in order to achieve the proposed learning outcomes. This is the case of the seminar presented in this communication. The heterogeneity of the training base of the attending students, as well as the singularity of it makes it necessary to use material developed in Online Teaching by the authors so that the students can understand the basic concepts of working pumps working as a turbine, Then, in the face-to-face phase, successfully carry out the proposed activities.
\end{abstract}

Keywords: Docencia en red, Polimedia; Hydraulic Engineering; Pumps operating as turbines; MUIHMA

\begin{abstract}
Resumen
El desarrollo de seminarios dentro del Máster Universitario en Ingenieria Hidráulica y Medio Ambiente (MUIHMA contribuyen a una formación complementaria transversal a la especialidad que desarrolla el alumno. El carácter intensivo en cuanto a tiempo de formación hace que el profesor deba considerar metodologías activas de formación por parte de alumnado para poder alcanzar los resultados de aprendizaje propuestos. Este es el caso del seminario presentado en esta comunicación. La heterogeneidad de la base de formación de los alumnos asistentes así como la singularidad del mismo hace necesario el empleo de material desarrollado en Docencia en Red por parte de los autores para que los alumnos puedan comprender los conceptos básicos de funcionamiento de bombas trabajando como turbina, para después en la fase presencial llevar a cabo con éxito las actividades propuestas.
\end{abstract}

Palabras clave: Docencia en Red; Polimedia; Ingeniería Hidráulica; Bombas operando como turbinas; MUIHMA

\section{Introducción}

El establecimiento del Espacio Europeo de Educación superior trae muchas implicaciones para los estudios impartidos en las titulaciones españolas. Con posterioridad, la aplicación en 
la UPV del programa, para aplicación de las competencias transversales (UPV, 2015), conlleva un giro copernicano, pasando del profesor como centro del aprendizaje, al alumno como sujeto activo del mismo. Particularmente, el aprendizaje autónomo y continuado es un ejemplo de cómo el alumno tiene que convertirse en protagonista de su propio aprendizaje.

De entre las estrategias que se tienen para conseguir este propósito y particularmente en últimos cursos de la formación de un alumno con un alto grado de madurez en máster, se encuentra la impartición de seminarios (Vela Sánchez, 2012; De Miguel et al, 2005). Un seminario es una estrategia de aprendizaje para el alumno que busca la profundización en un aspecto determinado de una asignatura o materia (Pérez Madrid, 2010). El seminario debe diferenciarse del curso clásico de impartición (o más bien aprendizaje) de una materia, porque este debe centrarse en la discusión y el análisis de un aspecto complementario de los contenidos presentados en la materia en sí (Arias Trujillo 2013). Asimismo, a juicio de los autores de la presente comunicación, un seminario en que el alumno puede elegir entre varias opciones, se prepara con anterioridad y se interesa por los contenidos ya a nivel de máster. Este corto periodo de formación es una ocasión única para la especialización de la formación de este alumno, ya casi dispuesto a incorporarse al mundo laboral.

Debe tenerse en cuenta que el seminario es una ocasión en que el alumno, tiene la oportunidad de aprender e investigar al mismo tiempo, por lo que es especialmente interesante en estudios a nivel de máster, que además tienen la opción de alentar a los alumnos a continuar con su formación doctoral en caso de que así lo deseen. Por otra parte, dado que se forman grupos pequeños muy interesados en aspectos concretos de las materias que desean ser profundizados por los alumnos, el trabajo en equipo es fundamental, por lo que la practicidad de los mismos alenta a los estudiantes a organizar sus trabajos en grupos, potenciando asimismo esta competencia tan importante en su futuro laboral, la organización a través de "pequeño grupo" muy interesado en tema frente al "gran grupo" involucrado en contenidos generalistas es la característica del seminario como estrategia docente universitaria (Malavé, 2010). De otro lado, los seminarios deben ser breves, concentrados y permitiendo al alumno realizar una búsqueda de contenidos en momentos anteriores o posteriores a la impartición de la materia que les motive y facilite su evaluación. En este sentido, el aprendizaje autónomo a través de las herramientas on-line que el profesor pueda facilitar al alumno (o este encontrar por sus medios) juegan un papel fundamental en la formación especializada que se espera de alumnos de últimas materias de máster.

Este es el caso del conjunto de seminarios ofertados en el Máster de Ingeniería Hidráulica y Medio ambiente, del que el Departamento de Ingeniería Hidráulica y Medio Ambiente de la UPV es Entidad Responsable de Titulo.

El Máster Universitario en Ingeniería Hidráulica y Medio Ambiente (MUIHMA) comprende dos períodos de docencia. Estos periodos son dos cuatrimestres (que abarcan un curso completo), más un período para la realización del trabajo fin de máster (TFM). El total de créditos son 60+15 créditos (UPV (MUIHMA), 2019), estableciéndose un máximo de 45 alumnos para el curso 2017/2018. No obstante, el número de matriculados podría haber sido mucho mayor, si se tiene en cuenta la lista de espera de alumnos de este año. 


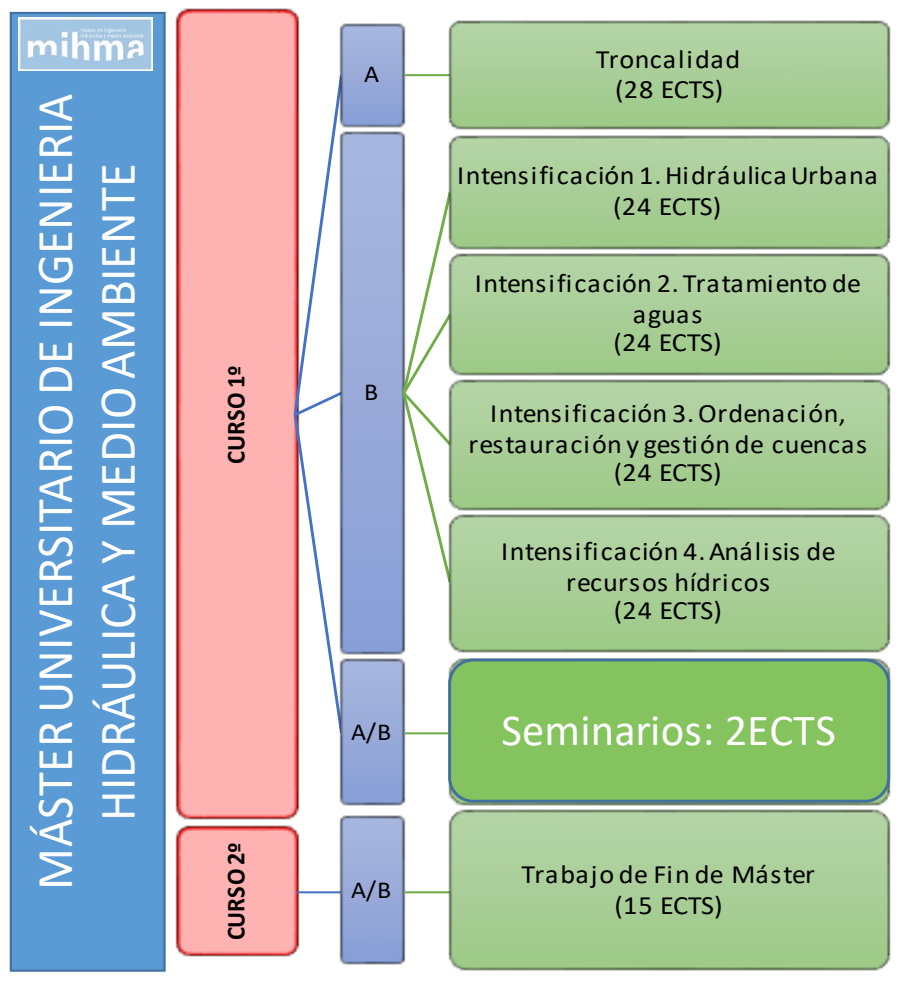

Figura 1. Estructura del MIHMA

La troncalidad de este máster (Cuatrimestre A) comprende materias en relación con la ingeniería hidráulica y calidad de aguas; mientras que cada una de las cuatro intensificaciones que se imparten comprenden las diferentes materias referentes a cada uno de los módulos o intensificaciones. El MUIHMA es un máster bien reconocido, que ha adquirido la acreditación ANECA+ y el sello de calidad internacional EUR-ACE®, desde 2016. El prestigio internacional del título es notorio, con una presencia internacional de estudiantes de más del $50 \%$ del alumnado y con mucha demanda entre las mejores universidades de habla hispana.

Como puede observarse en la Figura 1, los seminarios son una oferta permanente y amplia que puede cursarse en cualquiera de los dos cuatrimestres, ofreciendo al alumno la posibilidad de ampliar sus conocimientos en aspectos que no pueden ser abordados en ninguna de las asignaturas troncales, con gran éxito de propuesta y afluencia. La presente contribución se centra en la experiencia de los autores con el seminario: "Bombas funcionando como turbinas: principios generales", propuesto en los últimos cursos en el presente máster. 


\section{Objetivos}

La presente contribución tiene por objetivo analizar la estrategia docente del seminario "Bombas funcionando como turbinas: principios generales", propuesto en los últimos cursos en el Máster Universitario de Ingeniería Hidráulica y Medio Ambiente de la UPV. Este seminario viene a complementar particularmente los estudios en la intensificación de Hidráulica Urbana de dicho máster (Figura 1). Cómo objetivo, la comunicación muestra que el uso de polimedias pueden contribuir para formación complementaria antes, durante y después del seminario.

\section{Desarrollo de la Innovación}

La formación recibida por los alumnos del MUIHMA de la UPV en términos de utilización de las máquinas hidráulicas en redes de distribución de agua han sido impartidos en la asignatura "Hidráulica Avanzada" cursada de forma troncal por todos ellos en Cuatrimestre A. La impartición de esta asignatura ha despertado en los alumnos un interés por aspectos de eficiencia energética y análisis de aspectos de potencia hidráulica en las redes, que invita a la selección del presente seminario cuando se presenta el mismo como una estrategia de recuperación energética en las redes en puntos en que la energía se "desperdicia" a través de válvulas y puede ser recuperada con maquinaria sencilla que los alumnos ya conocen.

En este caso se presenta un seminario que tiene cinco horas presenciales. En este tiempo, se realiza un análisis participativo con los alumnos sobre los conceptos de maquinaria hidráulica que los alumnos ya conocen. Además, se profundiza de forma cooperativa sobre el funcionamiento de las bombas que los alumnos han aprendido en su funcionamiento directo, infiriendo cómo será el comportamiento inverso, cuando se instalen en la red funcionando como turbinas.

El desarrollo de estas horas presenciales se realiza a través del diálogo con los alumnos y del análisis del problema a través de un cuestionario que profundiza en el conocimiento de aspectos propios de este funcionamiento inverso, de la selección de las máquinas para el funcionamiento en turbinado y del conocimiento de la energía teórica recuperable en las redes. Se propone para los alumnos un taller que tienen que realizar a partir del conocimiento avanzado sobre los aspectos impartidos en el seminario. Durante las cinco horas el debate profesorado-alumnado está abierto y de forma continua se desarrollan apelaciones o interpretaciones de soluciones o problemáticas. Este debate tiene un gran enriquecimiento ya que el alumnado suele proceder de países diferentes a España, lo que pone de manifiesto la aplicación de la solución propuesta en el seminario en diferentes países con un nivel de desarrollo diferente.

El éxito de un seminario se basa en que los alumnos realicen una preparación autónoma con anterioridad. Es importante que se profundice con respecto a la materia avanzada que se va a impartir para que el desarrollo de las clases de forma colaborativa basada en la implementación de talleres y cuestiones sea productivo en las horas de aula. Se pone para ello a disposición del alumnado los objetos de aprendizaje realizados por los autores del 
presente trabajo, que ayudan al conocimiento profundo de los aspectos desarrollados en las horas presenciales del seminario que se describe.

El objetivo es que el alumno pueda interrelacionar los conceptos impartidos en asignaturas vistas en grado de nivel básico (e.g., mecánica de fluidos, máquinas hidráulicas), con asignaturas vistas en la troncalidad y especialidad del máster (e.g., hidráulica avanzada, eficiencia energética en redes) con conceptos ligados a la sostenibilidad del medio ambiente (Figura 2). El hecho de haber cursado estas asignaturas en otros años, o proceder de másteres o especialidades que no profundicen en algún tema en concreto, hace que el uso de objetos y módulos de aprendizaje sean cruciales en la formación transversal de alumno para que pueda alcanzar los resultados de aprendizaje propuestos en el seminario.

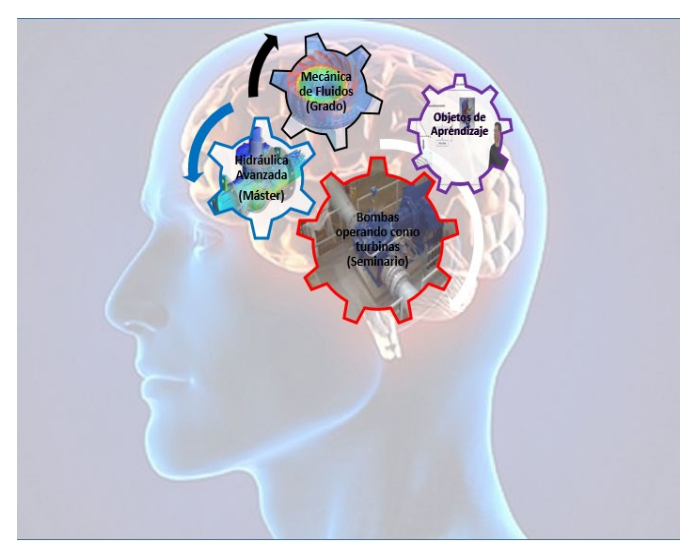

Figura 2. Ejemplo de interrelación de resultados de aprendizaje en asignaturas cursadas por el alumno

Los objetos de aprendizaje proceden del desarrollo de los autores de materiales en el marco del programa "Docencia en red" para preparación libre de videos cortos explicativos de aspectos concretos de una materia (López-Jiménez et al., 2016) y la combinación de esta preparación autónoma con el visionado de los videos y ciertos materiales especializados (fundamentalmente artículos de investigación ya publicados), es importante para la preparación y éxito del seminario.

El contenido genérico del material que se pone a disposición de los alumnos versa entorno a los siguiente temas:

- Aspectos de contextualización del uso de máquinas hidráulicas en las redes de distribución de agua. El resultado de aprendizaje establecido es "reconocer la tipología de máquinas hidráulicas que puede ser usada en sistemas de distribución".

- Aspectos de curvas motrices. El resultado de aprendizaje establecido es "conocer las curvas ideales, teóricas y reales de una máquina hidráulica operando como bomba y como turbina".

- Aspectos de funcionamiento y regulación. Este contenido tiene como resultado de aprendizaje "Definir los diferentes sistemas de regulación de una máquina operando como turbina en función del caudal". 
- Aspectos de Optimización. Finalmente, se establece un resultado de aprendizaje donde el alumno debe "conocer la necesidad de algoritmos de optimización en función de diferentes funciones objetivo".

\section{Resultados}

Cada curso existe alrededor de una treintena de seminarios ofertados para los 45 alumnos del MUIHMA. Algunos de ellos se activan y desactivan cada curso, de forma que la competencia natural por hacer los seminarios atractivos a los alumnos es importante. Asimismo, los alumnos pueden acudir a los seminarios participando en los mismos, más allá de estar matriculados oficialmente, puesto que son abiertos a todos los alumnos del máster en las sucesivas promociones.

Esto hace que la presencia de alumnos sea muy dispar entre todos los seminarios ofertados (en total existen 34 seminarios este curso 2018/2019), siendo este uno de los que mantiene la atención del alumnado con más de seis participantes (teniendo en cuenta que son 45 alumnos y que se inscriben en un máximo de 2 seminarios) en todas las ediciones en que se ha presentado (desde el curso académico 2016/17). Este número de alumnos ha permitido un trabajo colaborativo adecuado y los resultados de la evaluación a través del cuestionario final y el taller han sido altamente satisfactorios.

Por otra parte, para el éxito del seminario es muy importante un aprendizaje autónomo que sea realizado previamente a las horas presenciales. Este aprendizaje conecta los contenidos recibidos por los alumnos en asignaturas troncales del primer trimestre del MUIHMA con el seminario, tradicionalmente impartido en el segundo semestre. Entre ellos se oferta un número importante de objetos de aprendizaje en relaciopn con los aspectos tratados en el citado seminario y descritos anteriormente. Los objetos de aprendizaje desarrollados por los autores, entre otras cosas para motivar a los alumnos a profundizar en el tema que nos ocupa y a realizar un aprendizaje autónomo previo, son altamente consultados. Bien es verdad que no podemos seleccionar el número de consultas realizadas por estos alumnos en particular, pero al ser objetos abiertos, puede apreciarse el interés que despierta esta temática para la consulta a través de docencia asíncrona en los objetos preparados en esta temática, tal como se indica en la Tabla 1.

La información de retroalimentación aportada por los alumnos durante la realización de los seminarios muestra la satisfación de los mismos por los recursos de Docencia en Red elaborado por los profesores que imparten el seminario.

No obstante, este uso puede verse reflejado en la Tabla 1 en el número de descargas que tiene contabilizados así como en la visitas de estos polimedias en el canal de Youtube. Aunque estas descargas no son todas de los seminarios impartidos, a través de las estádisticas de riunet, si se ha podido observar la consulta de los polimedias recomendados durante el espacio temporal que se ha impartido el seminario. 
Tabla 1. Consulta de los objetos de aprendizaje desarrollados por los autores a través del programa Docencia en Red para la preparación del presente seminario

\begin{tabular}{|c|c|c|}
\hline Materia & Nombre del Objeto de Aprendizaje & $\begin{array}{l}\text { Numeri de } \\
\text { descargas }\end{array}$ \\
\hline \multirow{4}{*}{$\begin{array}{l}\text { Aspectos de contextualización } \\
\text { del uso de máquinas } \\
\text { hidráulicas en las redes de } \\
\text { distribución de agua }\end{array}$} & La necesidad de la estación de bombeo & 136 \\
\hline & Clasificación de las máquinas hidráulicas & 1018 \\
\hline & $\begin{array}{l}\text { La necesidad de válvulas en las redes de } \\
\text { distribución de agua }\end{array}$ & 82 \\
\hline & El ciclo urbano del agua & 63 \\
\hline \multirow[t]{5}{*}{ Aspectos de curvas motrices } & $\begin{array}{l}\text { Consideraciones sobre rendimientos y potencias en } \\
\text { turbomáquinas. }\end{array}$ & 44 \\
\hline & $\begin{array}{l}\text { Diferencia entre las ALTURAS de Euler, teórica y } \\
\text { real aportada por una bomba }\end{array}$ & 172 \\
\hline & $\begin{array}{l}\text { Teoría general de máquinas hidráulicas: la bomba } \\
\text { en la estación de bombeo }\end{array}$ & 289 \\
\hline & $\begin{array}{l}\text { Turbobombas: el papel del rodete y su relación con } \\
\text { las curvas teóricas de las máquinas }\end{array}$ & 241 \\
\hline & Elementos eléctricos en las estaciones de bombeo & 165 \\
\hline \multirow[t]{6}{*}{$\begin{array}{l}\text { Aspectos de funcionamiento y } \\
\text { regulación }\end{array}$} & $\begin{array}{l}\text { Asociación de turbomáquinas en una instalación } \\
\text { de bombeo. }\end{array}$ & 21 \\
\hline & Cavitación en turbomáquinas & 511 \\
\hline & $\begin{array}{l}\text { Cambio de la curva motriz al cambiar la velocidad } \\
\text { de rotación de una máquina hidráulica }\end{array}$ & 118 \\
\hline & $\begin{array}{l}\text { Comparativa en la regulación de la curva motriz en } \\
\text { máquinas hidráulicas }\end{array}$ & 87 \\
\hline & $\begin{array}{l}\text { Regulación de máquinas hidráulicas. Principios } \\
\text { fundamentales }\end{array}$ & 156 \\
\hline & Las leyes de semejanza en bombas hidráulicas & 1718 \\
\hline
\end{tabular}

\section{Conclusiones}

El desarrollo durante estos tres años del seminario "Bombas trabajando como turbinas. Principios Generales" llevado a cabo por los autores de la comunicación ha puesto de manifiesto que el uso de recursos de Docencia en Red, fundamentalmente objetos de aprendizaje, son un engranaje fundamental para la mejora de la adquisición de los resultados de aprendizaje en el seminario propuesto. Este hecho se ha visto reflejado en que alumnos procedentes de diferentes países, escuelas de ingeniería y diferente especialidad dentro de MUIHMA han sido capaces de llevar a cabo con éxito el seminario, alcanzando los resultados de aprendizaje establecidos. Asimismo, el uso de estos objetos de aprendizaje ha permitido llevar una alineación vertical y transversal de los objetivos propuestos, relacionando conceptos de asignaturas cursadas tanto en grado como en máster por parte de los alumnos. 
Los seminarios apoyados con formación online como estrategia de aprendizaje autónomo en últimos cursos de Master: caso práctico en el MUIHMA-UPV

\section{Referencias}

ARIAS-TRUJILLO, J.; PORRAS, R. (2013). "Los seminarios de problemas como estrategia docente en las enseñanzas técnicas: Una experiencia aplicada a la Ingeniería del Terreno". Revista de Docencia Universitaria. REDU. Vol. 11, Número especial dedicado a Engineering Education, pp. 47-64. Recuperado el (fecha de consulta) en http://red-u.net

DE MiguEL, M., ALFARO, I., APODACA, P., ARIAS, J., GARCÍA, E., LOBATO, C. Y PÉREZ, A. (2005). "Modalidades de enseñanza centradas en el desarrollo de competencias. Orientaciones para promover el cambio metodológico en el espacio europeo de educación superior”. Oviedo: Universidad de Oviedo, Servicio de Publicaciones. Ministerio de Educación y Ciencia.

LÓPEZ JIMÉNEZ, PETRA AMPARO; PÉREZ-SÁNCHEZ, MODESTO (2016). "Los objetos de aprendizaje como conductores para favorecer el aprendizaje autónomo en el campo de la mecánica de fluidos". En:Congreso Nacional de Innovación Educativa y Docencia en Red = Congrés Nacional d'Innovació Educativa i Docència en Xarxa (IN-RED 2016). (53 - 60). Valencia, España: Editorial UPV.

MALAVÉ OSUNA, B. 2010. "Una propuesta de proyecto de innovación educativa: seminarios y tutorías; dos estrategias didácticas para la nueva enseñanza de las disciplinas históricas". Revista Jurídica de Investigación e Innovación Educativa. 2: 101-110.

PÉREZ MADRID, F. 2010. "Movilidad y enseñanza tutorial: participación de estudiantes en seminarios y reuniones científicas con profesores universitarios". Innovación educativa en Derecho constitucional. Recursos, reflexiones y experiencias de los docentes, Lorenzo Cotino Hueso, Miguel Angel Presno (Coords.), Valencia: Universidad de Valencia, 241-244.

UPV (2015). Universitat Politècnica de València. "Institutional project of the generic outcomes". https://www.upv.es/entidades/ICE/info/Proyecto_Institucional_CT.pdf [Consultado 05/03/2018]

UPV. 2019. Máster Universitario en Ingenieria Hidráulica y Medio Ambiente. en marzo de 2019 en: https://www.upv.es/titulaciones/MUIHMA/ [Consultado marzo de 2019]

VELA SÁNCHEZ, A. (2012) "El seminario-control: Una manera de incentivar al alumnado". Revista UPO Innova. Vol I. Pp 518 a 529. 